\title{
The Critical Role of p38 MAP Kinase in T Cell HIV-1 Replication
}

\author{
Pamela S. Cohen, ${ }^{1}$ Helena Schmidtmayerova, ${ }^{1,4}$ \\ Jameel Dennis, ${ }^{1,2}$ Larisa Dubrovsky, ${ }^{1}$ Barbara Sherry, ${ }^{1}$ \\ Haichao Wang, ${ }^{1,3}$ Michael Bukrinsky, ${ }^{1}$ and Kevin J. Tracey, ${ }^{1,2}$ \\ ${ }^{1}$ The Picower Institute for Medical Research, Manhasset, New York, \\ U.S.A., ${ }^{2}$ Laboratory of Biomedical Science, Department of Surgery, \\ and ${ }^{3}$ Department of Emergency Medicine, North Shore University \\ Hospital, Manhasset, New York, U.S.A., ${ }^{4}$ Institute of Virology, \\ Slovak Academy of Sciences, Bratislava, Slovakia
}

\begin{abstract}
Background: Replication of HIV-1 in human T lymphocytes requires the activation of host cellular proteins. This study identifies p38 mitogen-activated protein kinase (MAPK) as one such kinase necessary for HIV-1 replication in $\mathrm{T}$ cells.

Materials and Methods: Primary human T lymphocytes were infected with the LAI strain of HIV-1 and Jurkat cells were infected with the RF strain of HIV-1. HIV replication was measured by reverse transcriptase activity. Cellular expression of endogenous p38 MAPK protein was analyzed using immunoprecipitation. Blockade of p38 MAPK expression was achieved using antisense oligonucleotides to p38 MAPK and the guanylhydrazone compound CNI-1493, an inhibitor of p38 MAPK activation.

Results: HIV-1 infection of both primary human T lymphocytes and a $\mathrm{T}$ cell line rapidly activated the cellular
\end{abstract}

p38 MAPK pathway, which remained activated for the duration of the culture. Addition of phosphothioated antisense oligonucleotides to $\mathrm{p} 38$ MAPK specifically inhibited viral replication. Blockade of p38 MAPK activation by addition of CNI-1493 also inhibited HIV-1 viral replication of primary $\mathrm{T}$ lymphocytes in a dose- and time-dependent manner. Stimulation of p38 MAPK activation did not occur with the addition of heat-inactivated virus, suggesting that viral internalization, and not just membrane binding, is necessary for p38 MAPK activation.

Conclusions: These results indicate that activation of the p38 MAPK cascade is critical for HIV-1 replication in primary $\mathrm{T}$ lymphocytes, and that blockade of this signal transduction pathway may be a novel therapeutic approach to the treatment of HIV-1 infection.

\section{INTRODUCTION}

HIV-1 infection has attained pandemic proportions. Combination therapies that target enzymes encoded by the virus, such as reverse transcriptase or protease, have shown promise in the treatment of this disease (1-4). Advances in understanding the pathogenesis of HIV-1 infection suggest that it may also be plausible to develop therapeutics for HIV-1 infection that target host protein cofactors. Such an approach would avoid the emergence of drug-resistant strains, a

Address correspondence and reprint requests to: Kevin J. Tracey, 350 Community Drive, Picower Institute for Medical Research, Manhasset, NY 11030, U.S.A. Tel: 516-5629476; email: ktracey@picower.edu. potential problem in every current anti-HIV-1 therapy directed against viral protein targets. One class of host proteins that participates in the regulation of HIV-l infectivity is transcription factors such as NF- $\kappa \mathrm{B}$, which stimulates viral gene expression through the long terminal repeat (LTR) of HIV-1 (5-7).

Proteins involved in signal transduction pathways represent another class of host protein targets. The serine/threonine kinase p38 MAPK, a member of the mitogen-activated protein (MAP) kinase superfamily of signal transduction molecules $(8-10)$, was recently implicated in the mediation of HIV-l infection in macrophages $(11,12)$. In the latently infected Ul promonocytic 
cell line, activation of p38 MAP kinase by tumor necrosis factor (TNF) and interleukin-1 (IL-1) caused reactivation of HIV-1 replication (11). It was further suggested that the molecular basis for this viral activation is due to p38 MAPKmediated enhancement of HIV-1-directed LTRtranscription (12).

Although p38 MAPK activation has also been implicated in signaling proinflammatory cytokine production in monocytes and macrophages, less is known about the role of p38 activation in T lymphocytes. p38 MAPK activation has been observed in lymphocytes during early intrathymic signaling, and is essential for the differentiation and repertoire selection of $\mathrm{T}$ cells during development (13). Although p38 MAP kinase activation has been implicated in HIV-1 infection of macrophages, (11), it is not known whether it is also required for HIV-1 infection of $\mathrm{T}$ lymphocytes. In this report, we demonstrate that p38 MAPK is activated in both primary $T$ lymphocytes and in a T cell line following HIV-1 infection, and this activation is required for HIV-1 replication in T lymphocytes.

\section{MATERIALS AND METHODS}

\section{Cell Culture and HIV Infection}

T lymphocytes were prepared by Ficoll-Hypaque separation of whole blood, depletion of monocytes by adherence, and activation with PHA $(5 \mu \mathrm{g} / \mathrm{ml})$ for 3 days in RPMI $1640+10 \%$ FCS + L-glutamine + antibiotics (penicillin, streptomycin). Cells were infected with $2.6 \times 10^{4} \mathrm{cpm} / \mathrm{ml}$ reverse transcription (RT) activity of HIV-1 LAI stock prepared on peripheral blood mononuclear cells (PBMCs). After a $2-\mathrm{hr}$ incubation at $37^{\circ} \mathrm{C}$, cells were washed and resuspended in fresh medium containing $20 \mathrm{U} / \mathrm{ml}$ of IL-2 (BoehringerMannheim, Indianapolis, IN). Virus was heat inactivated by incubation for $2 \mathrm{hr}$ at $56^{\circ} \mathrm{C}$. Where used, CNI-1493 (15), was added l hr prior to addition of virus, and cells refed with fresh media containing this agent every 3 days.

Jurkat cells (ATCC, Rockville, MD) were infected with the RF strain of HIV-1 as follows. Cells were infected with $2 \times 10^{5} \mathrm{cpm} / \mathrm{ml} \mathrm{RT}$ activity of HIV-1 RF stock prepared on PBMCs, and cell lysates were harvested after one-half $\mathrm{hr}$ for immunoprecipitation as described below.

For experiments using oligonucleotides, $\mathrm{T}$ lymphocytes were infected with HIV-1 $\mathrm{L}_{\text {LAI }}$ in the presence of $1 \mu \mathrm{M}$ 18-mer oligonucleotides. Cells were refed every 3-4 days with media containing fresh oligomer. At various time points after infection, supernatants were collected for RT assay and cells were harvested for total and phospho-p38 MAPK analysis assayed by Western blot and immunoprecipitation.

RAW 264.7 cells (ATCC, Rockville, MD) grown in RPMI $+10 \%$ FCS + antibiotics (PS) were pretreated with varying concentrations of CNI-1493 $1 \mathrm{hr}$ prior to addition of lipopolysaccharide (LPS) (Escherichia coli 0111:B4 $100 \mathrm{ng} /$ ml; Sigma Chemical Co., St Louis, MO). Cell lysates were prepared for immunoprecipitation and immunoblotting $15 \mathrm{~min}$ after administration of LPS.

Cells were assayed for viability using $0.04 \%$ Trypan blue stain (Gibco/BRL, Grand Island, NY).

\section{p38 MAPK Immunoprecipitation and Western Blotting}

At various time points after infection, cell lysates were prepared from $10^{7}$ cells/condition in lysis buffer $(0.05 \mathrm{M}$ Tris- $\mathrm{HCl}, 1 \% \mathrm{NP}-40,0.25 \% \mathrm{Na}$ deoxycholate, $150 \mathrm{mM} \mathrm{NaCl}, 1 \mathrm{mM}$ EDTA, $1 \mathrm{mM}$ $\mathrm{Na}_{3} \mathrm{VO}_{4}, 1 \mathrm{mM}$ NaF [Sigma Chemical Co.]). Equal amounts $(300 \mu \mathrm{g})$ of cell lysate were immunoprecipitated with the anti-phosphotyrosine antibody 4G10 (UBI, Lake Placid, NY) prebound to protein A-agarose beads (Sigma Chemical Co.), washed three times with lysis buffer, resolved on SDS-PAGE (12\%), and subjected to Western blotting using a 1:1000 dilution of rabbit polyclonal antibody prepared against the C-20 peptides of human p38 MAPK (Santa Cruz Biotechnology, Santa Cruz, CA). Binding to antibody was detected using enhanced chemiluminescence (Amersham International PLC, Arlington Heights, IL). p38 MAPK protein was measured using the densitometry program NIH image 1.59 .

Western blots of tyrosine phosphorylated proteins in whole cell lysates were probed using the antibody $4 \mathrm{Gl} 10$ as per manufacturer's recommendation.

\section{Antisense Oligonucleotides}

18-mer phosphothioate oligonucleotides coding for bp 324-341 in either the sense (GCAGG AGCTGAACAAGAC) or antisense (GTCTTGTT CAGCTCCTGC) direction were used on the basis of the sequence of human p38 MAPK (14). 

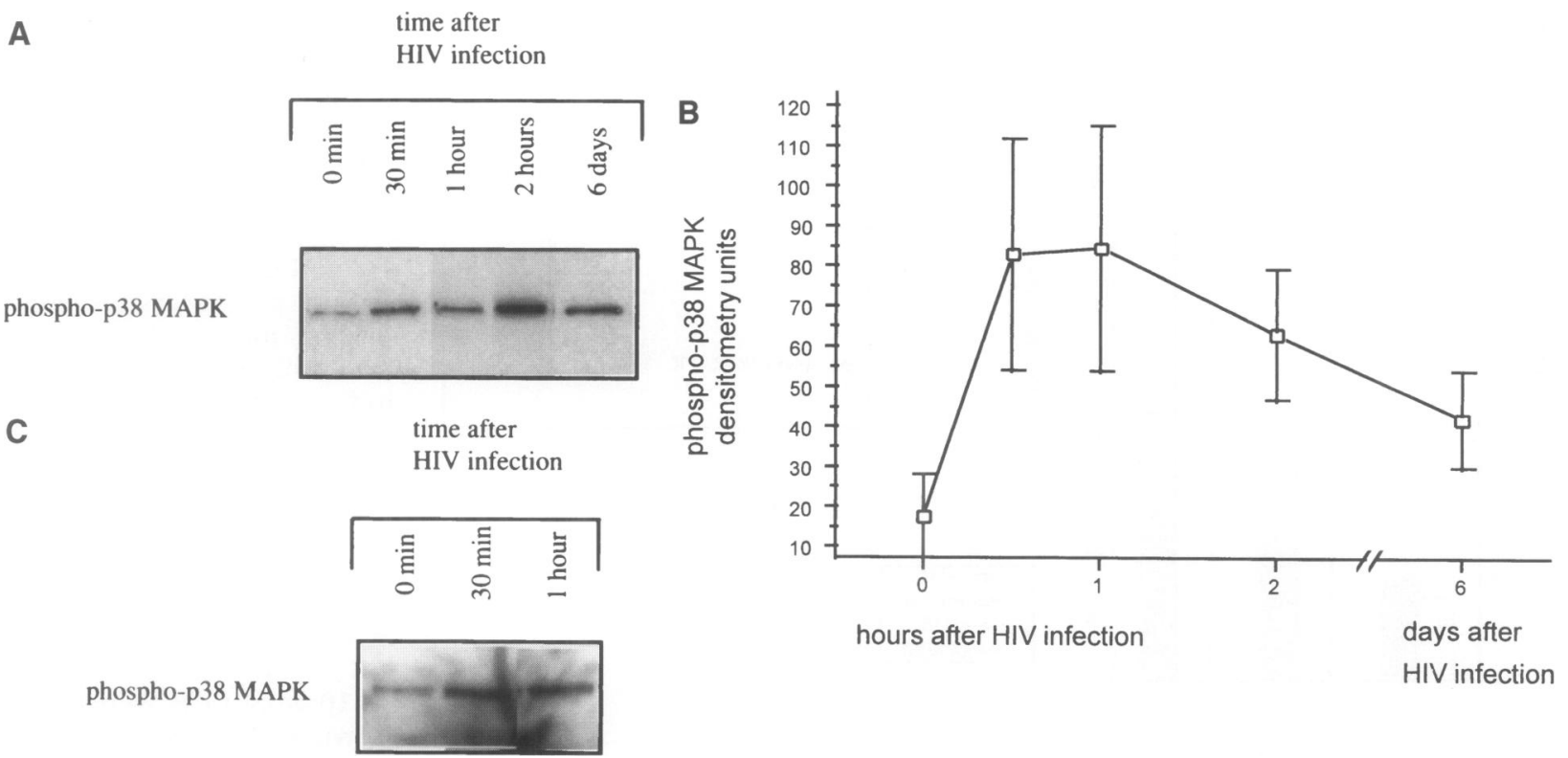

FIG. 1. Increased phosphorylation of p38 MAPK after primary infection of Tymphocytes with the LAI strain of HIV-1

Panel A depicts a representative protein immunoblot analysis of phospho-p38 MAPK levels in primary T lymphocytes in the absence or presence of HIV-1 $1_{\text {LAI }}$ at various time points after infection. Panel B shows the cumulative results of three experiments ( \pm standard error). Panel $C$ shows the results of a representative immunoblot analysis of phospho-p38 MAPK levels in the human $T$ cell line Jurkat in the absence or presence of HIV-1 $\mathrm{RF}_{\mathrm{RF}}$ at various time points after infection.

\section{RESULTS}

\section{Activation of p38 MAPK during Primary HIV-1 Infection of T Lymphocytes}

We measured p38 MAPK activation in primary $\mathrm{T}$ lymphocytes isolated from normal donors, stimulated with phytohemagglutinin (PHA) for 2-3 days, and then infected with the HIV- $\mathrm{l}_{\mathrm{LAI}}$ strain. p38 MAPK activation in these experiments was measured as a function of p38 MAPK phosphorylation assayed by immunoprecipitation. As early as $\mathbf{3 0}$ min postinfection, a rapid increase in the phosphorylation of p38 MAP kinase was observed in HIV-1-infected cells, as compared with control, uninfected cells (Fig. 1 A and B). Levels of p38 MAP kinase activity peaked at $2 \mathrm{hr}$ following introduction of virus into cultured $\mathrm{T}$ lymphocytes $(450 \%$ increase compared with uninfected cells; $p<.05$, Fig. 1B). The kinetics of early p38 MAPK activation in T lymphocytes as a consequence of HIV-1 infection correlated well with increases in p38 MAPK previously observed in macrophages following activation by other known stimuli of p38 MAPK, such as UV, TNF, and LPS (10). However, in primary $T$ cells infected with HIV, phospho-p38 MAPK levels did not return to the low baseline levels seen in uninfected cells (Fig. 1A and B), even after prolonged culture of $\mathrm{T}$ lymphocytes with virus for periods up to 6 days. This continued activation may be secondary to ongoing cycles of viral replication and cellular infection, as opposed to a single episode of stimuli as seen with the addition of UV, TNF, or LPS.

We next asked whether p38 MAPK is also activated in $\mathrm{T}$ cell lines infected with HIV. Jurkat cells were infected with the RF strain of HIV, then cell lysates collected at various intervals after infection and analyzed for phospho-p38 MAPK by immunoprecipitation. As was seen in primary $\mathrm{T}$ lymphocytes, levels of phospho-p38 MAPK rose substantially by $30 \mathrm{~min}$ after infection, and they remained elevated $1 \mathrm{hr}$ after infection (Fig. 1C). Thus p38 MAPK phosphorylation is stimulated in both primary $\mathrm{T}$ lymphocytes and $\mathrm{T}$ cell lines by HIV infection.

To determine whether binding of HIV-1 to a target cell is sufficient for induction of p 38 MAPK activation, we added heat-inactivated virus to primary $\mathrm{T}$ lymphocytes. At all time periods tested ( $30 \mathrm{~min}, 1,2$, and $4 \mathrm{hr}$ ), no stimulation of $\mathrm{p} 38$ 


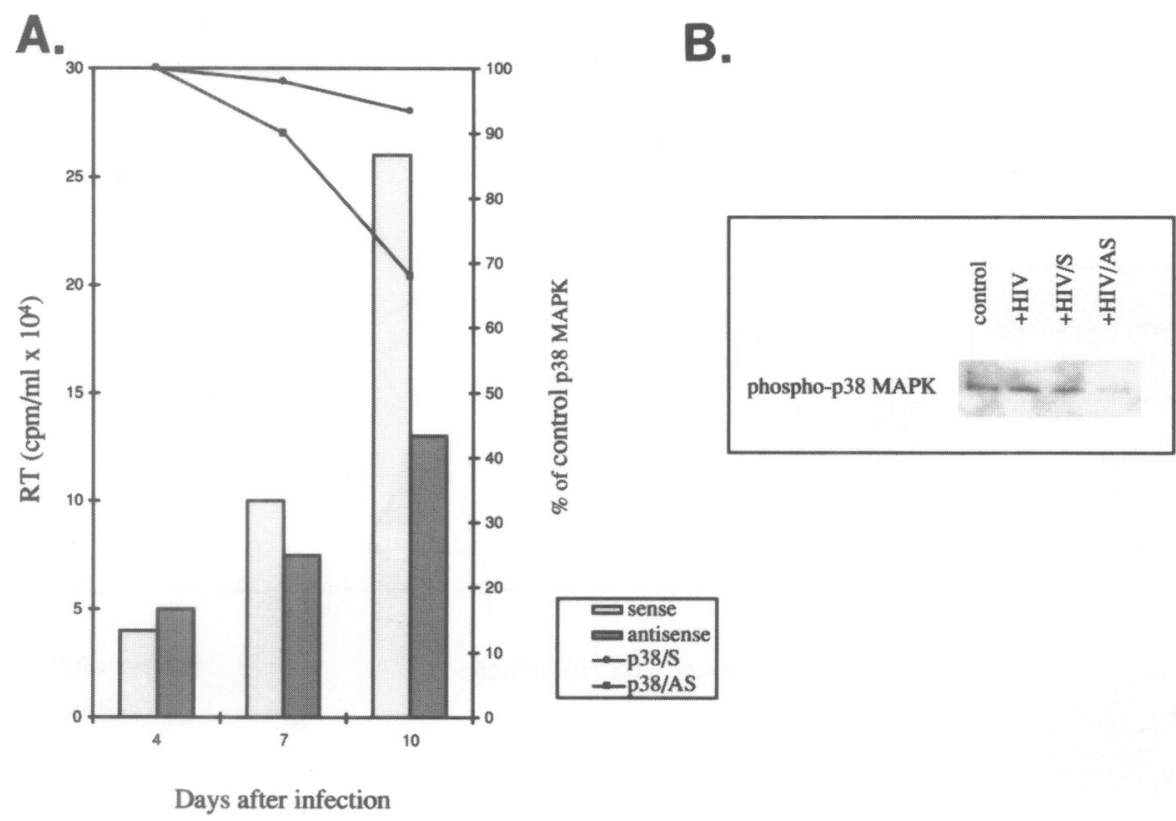

FIG. 2. Inhibition of HIV-1 replication in $T$ lymphocytes by phosphothioated antisense p38 MAPK oligonucleotides

(A) Shown here is the effect of sense and antisense p38MAPK oligonucleotides on total p38 MAPK protein levels as measured by Western immunoblot analysis, and its correlation with HIV-I replication as measured by RT. (B) Immunoblot analysis of phospho-p38 MAPK measured by immunoprecipitation of cell lysates treated with sense (S) or antisense (AS) phosphothioated p38 MAPK oligonucleotides for 10 days. Cells were grown as described in Materials and Methods.
MAPK was observed (data not shown). Thus binding of the virus to cellular receptors, which occurs with heat-inactivated as well as live virus, is not sufficient for p38 MAPK activation. Instead, fusion of the virus with the $\mathrm{T}$ cell membrane, or other steps downstream of membrane fusion, may trigger cellular p38 MAPK activation.

\section{p38 MAPK Antisense Oligonucleotides Inhibit Replication HIV-I}

Although these results suggested that activation of the p38 MAPK pathway is involved in HIV-1 replication, we wished to obtain evidence that activation of this signaling pathway was necessary for HIV-1 replication. We measured HIV-1 replication and p38 MAPK activity in T lymphocytes that were treated with phosphothioated antisense oligonucleotides against p38 MAP kinase. Antisense p38 MAPK oligomers, or control sense oligomers, were added to LAI-infected primary $\mathrm{T}$ lymphocytes simultaneously with virus, and then replenished every 3 days thereafter until Day 10. The p38 MAP kinase antisense oligonucleotides specifically decreased HIV-1 replication as compared with controls treated with sense oligomers (Fig. 2A). The decrease in reverse transcriptase levels correlated with the decrease in p38 MAPK protein levels on both Day 7 and Day 10 after infection (Fig. 2A). Because only the phosphorylated form of $\mathrm{p} 38$
MAPK is active, we also looked at levels of phospho-p38 MAPK in cell lysates after antisense oligonucleotide treatment. Interestingly, the decrease in phospho-p38 MAPK (70\%, Fig. 2B) was much more dramatic at Day 10 than the decrease in total p38 MAPK (32\%, Fig. 2A). Because suppression of $\mathrm{p} 38 \mathrm{MAPK}$ protein synthesis by antisense oligonucleotides inhibited HIV-1 replication in primary $\mathrm{T}$ lymphocytes, these results provide direct evidence that p38 MAPK activation is required for viral replication.

\section{CNI-1493 Inhibits HIV-1 Infection of T Cells via Inhibition of p38 MAPK Activation}

We recently developed a tetravalent guanylhydrazone compound (CNI-1493) as an effective inhibitor of proinflammatory cytokine synthesis (15-17). When CNI-1493 was added to the murine cell line RAW 264.7, it effectively suppressed the activation of p38 MAPK stimulated by LPS (Fig. 3A). Pharmacological concentrations of CNI-1493 reduced p38 MAPK activation in these cells to levels that were comparable to unstimulated controls (Fig. 3A). We therefore investigated whether this compound could inhibit HIV-1 replication in primary $\mathrm{T}$ cells. We measured p38 MAPK activation and viral replication in primary human $\mathrm{T}$ lymphocytes infected with the LAI strain, and observed that CNI-1493 mediated suppression of p38 MAPK activation 


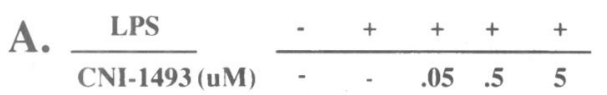
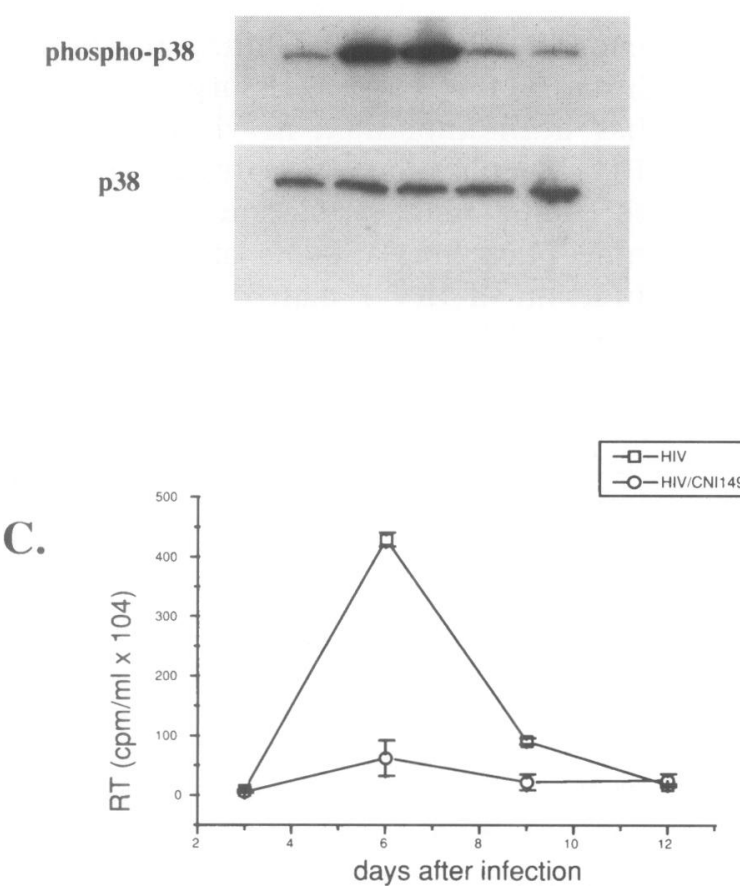

B.

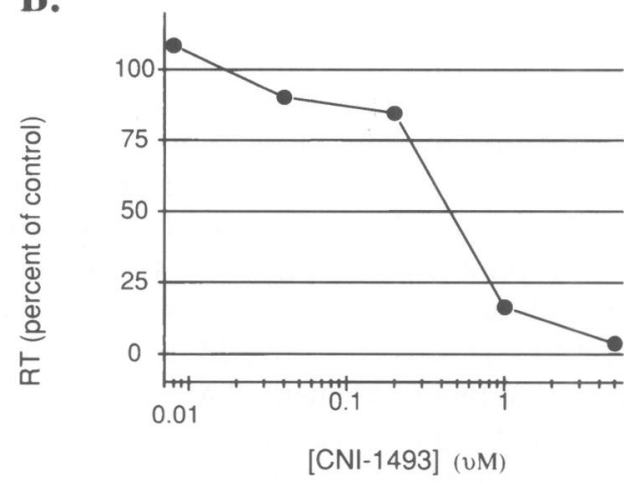

D.
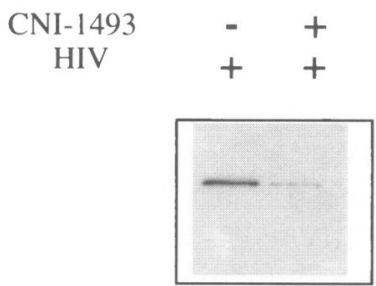

FIG. 3. Inhibition of HIV-1 replication in T lymphocytes by CNI-1493

(A) Immunoblot analysis of RAW 264.7 cells pretreated with varying concentrations of CNI-1493 $1 \mathrm{hr}$ prior to addition of LPS. Cell lysates were prepared for immunoprecipitation and immunoblotting (as described in Materials and Methods) 15 min after administration of LPS. (B-D) PHA-activated T lymphocytes were pretreated with control diluent or varying concentrations of CNI-1493 for $1 \mathrm{hr}$, infected with HIV-1 $1_{\mathrm{LAI}}$ as in Fig. 1, and cultured for 12 days. Cells were refed every 3 days with fresh media containing IL-2 \pm CNI-1493. Experiments were set up in duplicate to follow virus replication by measuring RT activity in the culture supernatants and to collect cell lysates at various time points for phospho-p38 MAPK immunoblotting. (B) Dose-dependent inhibition of RT by the addition of CNI-1493 on Day 6 of culture. (C) Time course of HIV-1 inhibition by $1 \mu \mathrm{M}$ CNI-1493. (D) Immunoblot of phospho-p38 MAPK expression in HIV-1-infected cells on Day 6 after infection and treatment with $1 \mu \mathrm{M}$ CNI-1493.

and HIV-1 replication (Fig. 3B, C, and D). The $50 \%$ inhibitory concentration $\left(\mathrm{IC}_{50}\right)$ of $\mathrm{CNI}-1493$ for suppressing viral replication was $0.5 \mu \mathrm{M}$, a concentration that was similar to that required for suppressing p38 MAPK activation in RAW cells (Fig. 3A). In time-course studies we observed that viral inhibition by CNI-1493 (1 $\mu \mathrm{M})$ persisted throughout the entire period of in vitro infection (Fig. 3C). Moreover, the magnitude of p38 MAPK suppression correlated with the suppression of HIV-1 replication (Fig. 3B, D).

The observed inhibitory effect of CNI-1493 on p38 MAPK activation after HIV-1 infection was not due to a toxic effect of CNI-1493 on T cells (Table 1). The possibility that the inhibitory
TABLE 1. Effect of CNI-1493 on primary $T$ lymphocyte viability

\begin{tabular}{cc}
\hline CNI-1493 ( $\boldsymbol{\mu M})$ & \% Viability \\
\hline 0.00 & 74.1 \\
0.05 & 92.6 \\
0.50 & 87.0 \\
5.00 & 71.4 \\
\hline
\end{tabular}

$2 \times 10^{4}$ primary $\mathrm{T}$ lymphocytes in $1 \mathrm{ml}$ of media were grown for 5 days in the presence of 20 units $/ \mathrm{ml}$ of IL-2 and varying concentrations of CNI-1493. Viable cells were assessed by the absence of Trypan blue uptake, and the total number of cells present was counted. 
effect of CNI-1493 on HIV-1 replication in T cells was due to a nonspecific inhibition of tyrosine phosphorylation was also evaluated. Western blots of cell lysates from $\mathrm{T}$ cells infected with HIV-1 in the presence or absence of CNI-1493 showed no significant difference in tyrosinephosphorylated proteins when analyzed using the anti-phosphotyrosine antibody $4 \mathrm{Gl} 0$ (data not shown). Finally, we have previously shown that CNI-1493 at levels $<20 \mu \mathrm{M}$ is not toxic when measured by metabolism of MTT or release of lactate dehydrogenase (LDH), and it does not affect levels of steady-state total protein or RNA synthesis (16).

\section{DISCUSSION}

Our results identify the p38 MAPK pathway as critical for HIV-1 replication in T lymphocytes. Infection of both primary $\mathrm{T}$ lymphocytes and a $\mathrm{T}$ cell line with HIV-1 causes a prompt and vigorous rise in the production of activated phosphorylated p38 MAPK. Inhibition of p38 MAPK protein using antisense phosphothioated oligonucleotides results in inhibition of HIV-1 replication in primary $\mathrm{T}$ lymphocytes. In addition, a similar inhibition of p38 MAPK mediated by CNI-1493 also inhibited HIV-1 replication in primary $\mathrm{T}$ lymphocytes.

Experiments presented here begin to elucidate the mechanism by which HIV-1 activates the $\mathrm{p} 38$ MAPK pathway in $\mathrm{T}$ cells. The addition of heat-inactivated virus did not appreciably raise the phospho-p38 levels in $T$ cells. Thus binding of the virus to membrane is not sufficient for p38 MAPK activation. Recent work by $\mathrm{Ku}$ mar et al. (18) shows that activation of the rashomolog CDC42HS, an upstream activator of p38 MAPK, is necessary for cytoskeletal and nuclear responses such as bacterial internalization via macropinocytosis that occur after infection of host cells with the bacterial pathogen Salmonella typhimurium. It is conceivable that a similar process occurs with membrane internalization of viral pathogens, activating CDC42HS, p38 MAPK, and other downstream activators. In addition, recent evidence from various investigators indicates that PAK65, another upstream activator of $\mathrm{p} 38$ MAPK that is itself activated by CDC42 and Racl, is also activated in both SIV and HIV infection (19-21).

The target of p38 MAPK activation and phosphorylation necessary for HIV-1 lymphocyte replication has yet to be identified. Activation of p38
MAP kinase is known to activate other proteins by phosphorylation, such as ATF-2, CHOP, HSP27, and Max (10,22-24). A number of HIV-1 accessory proteins also require phosphorylation in order to be activated during infection (25). For instance, the HIV-1 accessory protein Vif, which has been implicated in virion infectivity and HIV-1 replication, is activated by serine and threonine phosphorylation (26). Serine/threonine phosphorylation is also required for the function of HIV-1 matrix antigen in the early stages of infection (27). Activation of the p38 MAPK pathway has also been implicated in "cross talk" with other ras-MAPK pathways (28), including the MAPK/ERK1 pathway, which is important in regulating the transcription factor GABP. Activation of GABP can then participate in regulating HIV-1 Raf-RE (Raf-responsive elements), a region within the LTR promoter that is essential for regulation of HIV-1 expression (29). Lastly, the target of p38 MAPK critical for HIV replication may actually be via its effects on host TNF production in $T$ cells. TNF produced by $T$ cells is membrane-bound, rather than secreted, and little is known about the role of membranebound TNF in the regulation of HIV-1 infection of $\mathrm{T}$ cells.

The use of therapeutic agents to suppress p 38 MAPK activation could theoretically inhibit ongoing viral infection. Moreover, suppression of p38 MAPK activation inhibits TNF synthesis in monocytes and macrophages $(12,16,17)$, offering the additional advantage in HIV infection of preventing TNF-mediated systemic toxicity and reactivation of latent HIV-1-infected monocytic cells (30). Our observations that two independent methods of suppressing p38 MAPK activation (antisense oligonucleotides and CNI-1493) can both inhibit HIV-1 replication identify the p38 MAP kinase signal transduction pathway as critical for in vitro replication of HIV-1 in primary $\mathrm{T}$ lymphocytes. It is hoped that identification of this pathway in the pathogenesis of HIV-1 will facilitate the development of new therapeutic modalities.

\section{ACKNOWLEDGMENTS}

We thank Ms. Dee Prieto for excellent secretarial support. Part of this work was presented at the meeting Molecular Basis of Sepsis, October 8-11, 1996, at Woods Hole, MA. This work was supported by NIH grants DK49283 and NIH Al38245 and by institutional funding from The Picower Institute for Medical Research. 


\section{REFERENCES}

1. Collier AC, Coombs RW, Schoenfeld DA, et al. (1996) Treatment of human immunodeficiency virus infection with saquinavir, zidovudine, and zalcitabine. AIDS Clinical Trials Group. N. Engl. J. Med. 334: 10111017.

2. Eron JJ, Benoit SL, Jemsek J, et al. (1995) Treatment with lamivudine, zidovudine, or both in HIV-positive patients with 200 to 500 CD4+ cells per cubic millimeter. North American HIV Working Party [see comments]. N. Engl. J. Med. 333: 1662-1669.

3. Deeks S, Volberding P. (1995) An approach to antiretroviral treatment of HIV disease. Combined antiretroviral therapy: the emerging role. Hosp. Pract. (Off. Ed). 30 (Suppl 1): 23-31.

4. Fauci AS, Pantaleo G, Stanley S, Weissman D. (1996) Immunopathogenic mechanisms of HIV infection. Ann. Intern. Med. 124: 654663.

5. Heguy A, Stewart AA, Haley JD, Smith DE, Foulkes JG. (1995) Gene expression as a target for new drug discovery. Gene Expr. 4: 337-344.

6. Duh EJ, Maury WJ, Folks TM, Fauci AS, Rabson AB. (1989) Tumor necrosis factor alpha activates human immunodeficiency virus type 1 through induction of nuclear factor binding to the NF-kappa B sites in the long terminal repeat. Proc. Natl. Acad. Sci. U.S.A. 86: 5974-5978.

7. Osborn L, Kunkel S, Nabel GJ. (1989) Tumor necrosis factor alpha and interleukin 1 stimulate the human immunodeficiency virus enhancer by activation of the nuclear factor kappa B. Proc. Natl. Acad. Sci. U.S.A. 86: 2336-2340.

8. Han J, Lee J-D, Bibbs L, Ulevitch RJ. (1994) A MAP kinase targeted by endotoxin and hyperosmolarity in mammalian cells. Science 265: 808-811.

9. Lee JC, Laydon JT, McDonnell PC, et al. (1994) A protein kinase involved in the regulation of inflammatory cytokine biosynthesis. Nature 372: 739-746.

10. Raingeaud J, Gupta S, Rogers JS, et al. (1995) Pro-inflammatory cytokines and environmental stress cause p38 mitogen-activated protein kinase activation by dual phosphorylation on tyrosine and threonine. J. Biol. Chem. 270: 7420-7426.

11. Shapiro L, Dinarello CA. (1996) Specific in- hibition of p38 mitogen activated protein (MAP) kinase inhibits in vitro HIV replication in $\mathrm{Ul}$ cells induced by interleukin (IL)-1, tumor necrosis factor (TNF) or phorbol esters (PMA). Eur. Cytokine Netw. 7(3): 557.

12. Kumar S, Orsini $M$, Lee J, McDonnell $P$, Debouck C, Young P. (1996) Activation of the HIV LTR by UV, cytokines and high osmolarity requires CSBP/p38 activation. Eur. Cytokine Netw. 7(3): 558.

13. Sen J, Kapeller R, Fragoso R, Sen R, Zon LI, Burakoff SJ. (1996) Intrathymic signals in thymocytes are mediated by p38 mitogenactivated protein kinase. J. Immunol. 156: 4535-4538.

14. Han J, Richter B, Li Z, Kravchenko V, Ulevitch RJ. (1995) Molecular cloning of human p38 MAP kinase. Biochim. Biophys. Acta 1265: 224-227.

15. Bianchi $M$, Ulrich $P$, Bloom O, et al. (1995) An inhibitor of macrophage arginine transport and nitric oxide production (CNI-1493) prevents acute inflammation and endotoxin lethality. Mol. Med. 1: 254-266.

16. Bianchi $M$, Bloom $O$, Raabe $T$, et al. (1995) Suppression of proinflammatory cytokines in monocytes by a tetravalent guanylhydrazone. J. Exp. Med. 183: 927-936.

17. Cohen PS, Nakshatri H, Dennis J, et al. (1996) CNI-1493 inhibits monocyte/macrophage tumor necrosis factor by suppression of translation efficiency. Proc. Natl. Acad. Sci. U.S.A. 93: 3967-3971.

18. Kumar S, Orsini MJ, Lee JC, McDonnell PC, Debouck C, Young PR. (1996) Activation of the HIV-1 long terminal repeat by cytokines and environmental stress requires an active CSBP/p38 MAP kinase. J. Biol. Chem. (In Press).

19. Lu X, Wu X, Plemenitas A, et al. (1996) CDC42 and Racl are implicated in the activation of the Nef-associated kinase and replication of HIV-1. Curr. Biol. 6: 1677-1684.

20. Nunn MF, Marsh JW. (1997) Human immunodeficiency virus type 1 NEF associates with a member of the p21-activated kinase family. J. Virol. 70: 6157-6161.

21. Sawai ET, Khan IH, Montbriand PM, et al. (1996) Activation of PAK by HIV and SIV Nef: Importance for IADS in Rhesus macaque. Curr. Biol. 6: 1519-1527.

22. Batchvarova N, Wang XZ, Ron D. (1995) Inhibition of adipogenesis by the stress-in- 
duced protein CHOP (Gadd153). EMBO J. 14: 4654-4661.

23. Freshney NW, Rawlinson L, Guesdon F, et al. (1994) Interleukin-1 activates a novel protein kinase cascade that results in the phosphorylation of Hsp27. Cell 78: 10391049.

24. Zervos AS, Faccio L, Gatto JP, Kyriakis JM, Brent R. (1995) Mxi2, a mitogen-activated protein kinase that recognizes and phosphorylates Max protein. Proc. Natl. Acad. Sci. U.S.A. 92: 10531-10534.

25. Trono D. (1995) HIV accessory proteins: Leading roles for the supporting cast. Cell 82: 189-192.

26. Yang X, Goncalves J, Gabuzda D. (1996) Phosphorylation of Vif and its role in HIV-1 replication. J. Biol. Chem. 271: 10121-10129.
27. Heinzinger NK, Bukinsky MI, Haggerty SA, et al. (1994) The Vpr protein of human immunodeficiency virus type 1 influences nuclear localization of viral nucleic acids in nondividing host cells. Proc. Natl. Acad. Sci. U.S.A. 91: 7311-7315.

28. Xia Z, Dickens M, Raingeaud J, Davis RJ, Greenberg ME. (1995) Opposing effects of ERK and JNK-p38 MAP kinases on apoptosis. Science 270: 1326-1331.

29. Flory E, Hoffmeyer A, Smola U, Rapp UR, Bruder JT. (1996) Raf-1 kinase targets GAbinding protein in transcriptional regulation of the human immunodeficiency virus type 1 promoter. J. Virol. 70: 2260-2268.

30. Kaplan G, Moreira AL. (1994) TNF alpha regulation of HIVl: Biology and therapy. Res. Immunol. 145: 685-689.

Communicated by R. Bucala. Accepted March 28, 1997. 